\title{
Brechas de equidad en la evaluación censal de estudiantes 2007-2014: tres aproximaciones a su medición
}

\author{
César Guadalupe* \\ Universidad del Pacífico. Lima, Perú \\ Andrés Burga** \\ Universidad de Lima \\ y Universidad Peruana de Ciencias Aplicadas. Lima, Perú \\ Liliana Miranda*** \\ Ministerio de Educación. Lima, Perú \\ Luis Eduardo Castillo**** \\ Universidad del Pacífico. Lima, Perú
}

Recibido: 8 de junio de 2015 / Aprobado: 13 de julio de 2015

Este artículo discute la evidencia generada mediante la evaluación censal de estudiantes del Perú (aplicada en el segundo grado de educación primaria para medir desempeños en comprensión lectora y matemática) entre 2007 y 2014, con relación a brechas de equidad vinculadas al sexo de los estudiantes, la gestión de la escuela (estatal/no estatal); el área geográfica (urbana/rural); y su característica (escuelas completas/multigrados).

evaluación de aprendizajes / evaluación censal de estudiantes / brechas de equidad en aprendizajes

\section{Inequity Gaps in the 2007-2014 Student Assessment Census: Three Approaches to Measurement}

This article discusses the evidence produced by the census-based student assessment (conducted in grade two -primary education to measure performance in relation to reading comprehension and mathematics) between 2007 and 2014 in relation to equityrelated gaps regarding students' sex; school management type (State-run; Privately-run); location (urban/rural areas); and the manner in which the provision of instruction is organised (complete/multigrade schools).

learning assessment / student evaluation census / equity gaps in learning

Correos electrónicos:*A.GuadalupeM@up.edu.pe; **aburga@ulima.edu.pe; pcpsabur@upc.edu.pe; ***1miranda@minedu.gob.pe; ****LE.CastilloA@up.edu.pe 
La preocupación por la equidad en la educación no es un tema accesorio, sino uno que toca a la propia definición de la educación como derecho humano fundamental. Así, la preocupación por la equidad no solo es un posible elemento que contribuye, o no, a definir las políticas educativas, sino que es parte de cualquier definición comprensiva de lo que es un sistema educativo de calidad: no hay sistema educativo de calidad si no es equitativo (Unesco/ Santiago, 2007).

Esta preocupación ha estado permanentemente presente en la literatura educativa en general y forma parte de preocupaciones fundantes de los sistemas de evaluación de los aprendizajes. Por ejemplo, el sistema de evaluación de aprendizajes de Estados Unidos fue concebido en los años sesenta, en el marco de la legislación sobre derechos civiles; así, la medición de aprendizajes debía contribuir a constatar si el sistema educativo brindaba oportunidades educativas de modo equitativo a los diferentes segmentos de la población (Coleman, Campbell, Hobson, McPartland, Mood y Weinfeld, 1966).

Las mediciones estandarizadas de logros de aprendizaje de los estudiantes peruanos, tanto nacionales como internacionales, muestran progresos importantes que si bien parten de una situación particularmente precaria, no pueden ser desdeñados. En efecto, evaluaciones como el Programme for In- ternational Student Assessment (PISA) de la Organización para la Cooperación y Desarrollo Económicos (OCDE) (OECD, por sus siglas en inglés) muestran progresos sostenidos para el período 2001-2009-2012 en el terreno de la comprensión lectora (Ministerio de Educación, 2013); del mismo modo, las evaluaciones latinoamericanas de 2006 y 2013 (Unesco/Santiago, 2014) muestran progresos en lectura, matemática y ciencias. Finalmente, la Evaluación Censal de Estudiantes (ECE) también exhibe progresos importantes para el período 2007-2014.

Ahora bien, ¿en qué medida estas mejoras en los desempeños medios de nuestros estudiantes se han visto acompañadas de modificaciones en las diferencias que se observan entre distintas subpoblaciones? O, dicho de otra forma, ¿en qué medida este progreso medio ha ido acompañado de una mejora/deterioro/estancamiento en las brechas de equidad existentes entre nuestros estudiantes?

Este artículo aborda el tema a partir de la información generada anualmente a través de la evaluación censal de estudiantes (ECE) para el período 2007-2014. Para ello parte de una breve discusión, en la que se presentan las distintas medidas que pueden usarse para dar cuenta de estas brechas y explica por qué es necesario hacer un uso conjunto de más de una de ellas. Seguidamente, se presenta la evidencia con 
unos casos ilustrativos y una mirada sumaria de la totalidad de la información que está contendida en los anexos. A partir del análisis, el artículo termina identificando algunos patrones de comportamiento diferenciados en las brechas y presenta una serie de reflexiones finales acerca de los resultados y el alcance permitido por usar diferentes formas de aproximación al tema que son usados de modo conjunto.

\section{NOTAS PRELIMINARES}

La ECE es el esfuerzo de mayor alcance que se ha desarrollado en el país para conocer los niveles de logro académico obtenidos por los estudiantes de educación básica. Esta evaluación mide, desde 2007, competencias básicas en comprensión lectora y matemática en todas las escuelas primarias del país, con dos excepciones: las escuelas en las que se aplica un programa de educación bilingüe (donde se conduce una evaluación en cuarto grado que no es comparable con la anterior) y en las escuelas con menos de cinco estudiantes matriculados en segundo grado.

Asimismo, es importante considerar que la ECE comprende dos eventos estadísticos simultáneos: la evaluación censal propiamente dicha, y la administración de las pruebas en una "muestra de control".

Esto es muy importante, dado que las limitaciones logísticas propias de un operativo censal llevan a la necesidad de conducir un evento paralelo en condiciones de administración más controladas, que permitan obtener estimaciones nacionales robustas y validar los propios resultados del evento censal.

En este artículo se usará información de la muestra de control por ser la que produce estimaciones más robustas, ya que la mera agregación de la información censal (sin considerar la existencia de diferentes tasas de respuesta por estrato y año) puede contener sesgos producto del proceso de administración.

El uso de información muestral supone considerar que las estimaciones son computadas con un margen de error conocido. En todos los casos, se ha optado por hacer estimaciones con un $95 \%$ de certeza a partir de los errores estándar computados, considerando que se trata de un diseño muestral complejo.

De esta forma, las brechas estimadas son la distancia entre el límite inferior del intervalo de confianza de la estimación de cada indicador para el grupo en ventaja, y el límite superior del intervalo de confianza de la estimación de cada indicador para el grupo en desventaja.

Este procedimiento se explica porque la evidencia estadística existente (dados los márgenes de error propios de la información muestral) solo permite afirmar, con el nivel de confianza adoptado, la existencia de esta brecha, 
que es menor a la que se obtendría de simplemente comparar los estimadores muestrales (o los puntos medios de los intervalos de confianza) $)^{1}$.

Dado lo anterior, el análisis se enfocará en los estratos considerados para el diseño de la muestra de control de la ECE, es decir, sexo (varones y mujeres); área de residencia (escuelas ubicadas en conglomerados urbanos o en localidades rurales); gestión (escuelas estatales o no estatales); y lo que en el Perú se llama "característica" de la oferta educativa, es decir, si la experiencia de enseñanza-aprendizaje se organiza en grupos de un solo grado o de más de un grado (polidocencia completa o multigrado/unidocente).

Es imperativo señalar que la ECE brinda información muy importante pero limitada a dos aspectos de la experiencia educativa y de los aprendizajes esperados para los estudiantes peruanos. En ese sentido, es muy importante considerar que la ECE no mide todos los aspectos que debemos valorar, o que constituye una medida de la "calidad" de la educación. Del mismo modo, es importante considerar que los logros de aprendizaje de los estudiantes son explicados -como lo ha mostrado abundante literatura internacional y en el caso peruano Coleman et al., (1966); Cueto, Guerrero, León, Zapata y Freire (2013); Guadalupe y Villanueva (2013); Ministerio de Educación (2005) y Willms (2002) - no solo por la experiencia escolar sino también por factores extraescolares, de modo que la información de la ECE no es suficiente para identificar qué escuelas o grupos de escuelas son "mejores" que otras.

\section{Aproximaciones a la medición DE LAS BRECHAS DE EQUIDAD}

En este documento hemos considerado tres formas de medir brechas entre dos grupos de comparación, a partir del porcentaje de estudiantes ubicados en un nivel de desempeño dado ${ }^{2}$ :

a) Tamaño absoluto en, por ejemplo, puntos porcentuales. En este caso se computa, simplemente, la diferencia en los tamaños relativos de las fracciones de cada población comparada que cumplen con un criterio determinado (Kline, 2004). Si el porcentaje de estudiantes con desempeño satis-

1 Por cierto, la medición de las brechas así definidas también contiene un error de estimación. Este no ha sido presentado para evitar hacer aún más compleja la lectura del texto. Sin embargo, se llama la atención del lector sobre este tema, a efectos de hacer una lectura cuidadosa de los resultados presentados.

2 La ECE considera, además del porcentaje de estudiantes en cada nivel de desempeño, el promedio de la medida estimada a partir del escalamiento de los ítems usando el modelo Rasch (Bond y Fox, 2001). En el presente artículo únicamente se trabajará con la primera de las formas de reportar resultados ya que al ser la medida Rasch una escala de intervalo, solo se pueden trabajar brechas considerando la diferencia (perspectiva aditiva) y no cocientes, pues estos cambian al transformar linealmente la escala como puede hacerse con una medida estimada mediante cualquiera de los modelos Rasch (Wright y Linacre, 1987). 
factorio en el grupo 1 es de $35 \%$ y de $25 \%$ en el grupo 2, el tamaño de la brecha es igual a $35-25=10$ puntos porcentuales. La limitación más importante de esta medida es que no considera el valor relativo que representa una diferencia dada al no tomar en cuenta el valor absoluto de las magnitudes comparadas. Si en un grupo la condición observada (por ejemplo, desempeño satisfactorio) se observa en $4 \%$ de los casos y en otro en $2 \%$, la diferencia (dos puntos) sería equivalente a la existente entre valores de 98 y $96 \%$, cuando en el segundo caso dicha diferencia es muy pequeña en términos relativos y en el primero significa que la condición se cumple para el primer grupo en el doble de los casos que en el otro.

Lo mismo se aplica cuando se hace comparaciones entre dos momentos en el tiempo para ver la evolución de las diferencias. Por ejemplo, si el desempeño satisfactorio sube en el grupo 1 de 20 a $30 \%$ y en el segundo de 10 a $20 \%$ entre dos momentos en el tiempo, la brecha permanece del mismo tamaño (10 puntos porcentuales) aun cuando el segundo grupo experimentó un progreso del $100 \%$ (10 puntos sobre un punto de partida de 10 puntos porcentuales) y el primero solo de $50 \%$ (10 puntos sobre un punto de partida de 20 puntos porcentuales).

Tomar en cuenta la limitación de esta medida es particularmente importante en la medición de logros de aprendizaje, considerando que los cambios podrían ser cualitativamente distintos, dependiendo del punto de partida.

b) Dadas las limitaciones de la medida anterior, es también importante observar las diferencias relativas, que son computadas bajo la forma de un cociente de riesgo relativo ${ }^{3}$ o índice de paridad. El riesgo relativo o índice de paridad es calculado como el cociente entre el valor del indicador medido para un grupo y el valor del mismo indicador para un grupo de comparación (A'Court, Stevens y Heneghan, $2012)^{4}$. Así, un riesgo relativo o índice de paridad igual a uno indicaría que no hay diferencias entre el valor del indicador para los dos grupos; por otra parte, si el índice fuese mayor que uno indicaría que el evento o resultado es más común (y cuánto más común) en el grupo inicial (y menor en el grupo de comparación); y si fuese menor a uno, lo contrario.

3 El término riesgo relativo obedece a que es una forma de medición muy usada en el mundo de la epidemiología y la investigación médica. Así, usualmente se compara un grupo de "tratamiento" con uno de "control", de modo que se pueda dar cuenta si en el primer caso la exposición al tratamiento influye sobre el "riesgo" de, por ejemplo, contraer una enfermedad, tal y como se observa en el grupo de control.

4 Para el presente estudio, esto equivale a dividir el número de individuos que experimentaron el evento o resultado entre el tamaño de la muestra (Sistrom y Garvan, 2004). 
Por ejemplo, si se analiza el porcentaje de estudiantes con desempeño satisfactorio en comprensión lectora y se tuviese que el riesgo relativo o índice de paridad entre el grupo de estudiantes de escuelas urbanas $(20 \%)$ y escuelas rurales $(10 \%)$ es dos, esto indicaría que para un estudiante de escuela urbana es el doble de probable (que para los estudiantes rurales) que se encuentre dentro del nivel de desempeño satisfactorio. Del mismo modo, si el riesgo relativo fuese de 0,5 (como resultado de dividir, por ejemplo, 10/20), esto indicaría que la probabilidad de los estudiantes del primer grupo de mostrar un desempeño satisfactorio es la mitad de la observada entre los estudiantes del segundo grupo.

c) Junto a la medida anterior existe también una medida que compara la probabilidad de encontrarse en una condición dada en un grupo específico relativa a la probabilidad de no estarlo para el mismo grupo, con una medida similar para el grupo de comparación (Kline, 2004). Esta medida, que lamentablemente no tiene un nombre comúnmente aceptado en castellano ${ }^{5}$, es el Odds ratio.
Este es una medida relativa de efecto que compara los $o d d s^{6}$ de un evento para un grupo -de "tratamiento" en el caso de la investigación médica o cuando se evalúa intervenciones- y un grupo de referencia o "control" (Szumilas, 2010).

Si el odds ratio es igual a uno, las probabilidades de ocurrencia del evento son iguales en ambos grupos. Sin embargo, si el ratio fuese mayor a uno, el grupo de comparación o control tendría un odd menor de que el evento ocurra, lo que indicaría que en el primer grupo hay mayores probabilidades de ocurrencia; y si el ratio fuese menor a uno, lo inverso.

Por ejemplo, si se analiza el evento de estar dentro del nivel de desempeño satisfactorio en comprensión lectora, y se tuviese que el odd para los estudiantes de escuelas urbanas es $0,6 \mathrm{y}$ el odd de un estudiante de escuela rural es 0,4, entonces el odds ratio sería 1,5. Esto indicaría que para un estudiante, ceteris paribus, el odd de estar en el nivel de desempeño satisfactorio aumentan en un $50 \%$ si está en una escuela urbana en vez de una rural.

Dadas las consideraciones previas, en este artículo se hará uso de estas tres

5 Lo más cercano sería hablar de razón de chances.

6 Se entiende como odd al cociente de la probabilidad de que un evento ocurra y la probabilidad de que el evento no ocurra. Así, por ejemplo, si la probabilidad de que un alumno de escuela rural esté por encima del nivel satisfactorio es 0,2 , el $o d d$ sería $0,2 \div 0,8=1 / 4$, lo que se interpreta como que de cada alumno de escuela rural por encima del nivel satisfactorio, cuatro alumnos se encuentran por debajo de dicho nivel (las odds $-\mathrm{o}$ chances-de encontrarse en el nivel satisfactorio son de 1 a 4). 
medidas para el caso de los porcentajes de estudiantes en un determinado nivel de desempeño (el porcentaje de estudiantes por encima del nivel de desempeño satisfactorio y el porcentaje en el nivel de desempeño inicial).

\section{LO QUE LA EVIDENCIA MUESTRA PARA EL PERÍODO 2007-2014}

Esta sección se aboca a un análisis descriptivo simple de la información disponible gracias a las evaluaciones censales de estudiantes, y para ello se presenta, primero, a manera de ilustración, la evolución de las medidas antes descritas para el porcentaje de estudiantes con desempeño satisfactorio en comprensión lectora. Luego, para no sobrecargar el texto con todos los gráficos que serían posibles, se procederá a sintetizar toda la información disponible de las tres medidas, tanto para comprensión lectora como matemáticas, y para los niveles de desempeño satisfactorio e inicial (toda la información se incluye en la tabla 4 del anexo), mediante dos tablas resumen (tabla 1 y tabla 2).

Como se puede apreciar en la figura 1 relativo al porcentaje de estudiantes que logra desempeños satisfactorios en

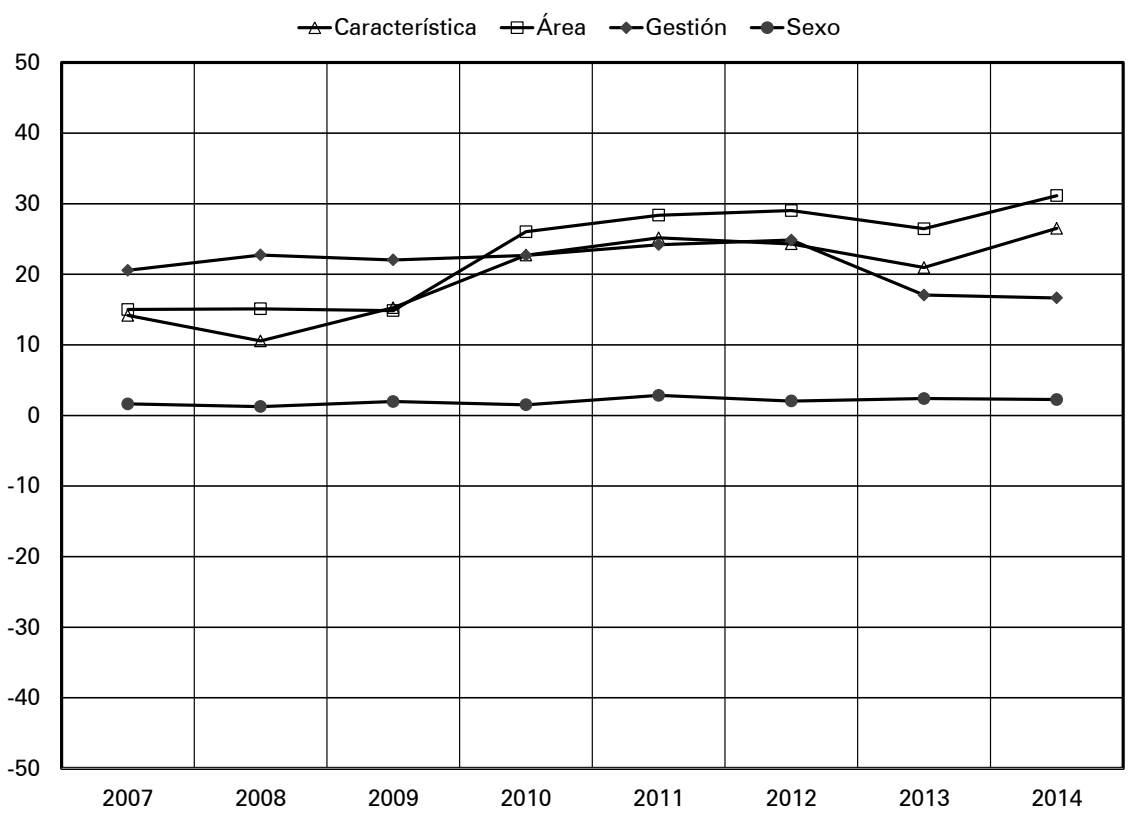

Figura 1. Distancia en puntos porcentuales entre los estudiantes de segundo grado que alcanzan el nivel de desempeño satisfactorio en comprensión lectora según estratos. Evaluación censal de estudiantes. Perú, 2007-2014.

Elaboración propia, a partir de la información disponible en http://umc.minedu.gob.pe/ (recuperada el 16 de marzo de 2015). 
comprensión lectora, las diferencias según el sexo de los estudiantes (a favor de las niñas) son de una magnitud mucho menor a las otras registradas, pero han mostrado una tendencia hacia la ampliación (pasando de 1,64 en el 2007 a 2,25 en el 2014; es decir, el incremento fue de 2,26 veces).

Por su parte, la brecha asociada a la gestión muestra una tendencia a la ampliación (de 20,6 puntos en 2007 a 24,9 puntos en 2012) para en los últimos dos años mostrar un comportamiento hacia la disminución (alcanza 16,7 puntos en 2014). Así, la brecha en puntos porcentuales observada en 2014 es un quinto menor que la de 2007 y un tercio menor que la registrada en $2012^{7}$.

La brecha por área (urbano/rural) muestra una tendencia marcada hacia la ampliación. Así, mientras que esta representaba 15 puntos porcentuales en 2007, ha ascendido a poco más del doble
(31,1 puntos) para el 2014, observándose el mayor salto entre 2009 y $2010^{8}$.

Por último, la información de acuerdo a la "característica" del proceso de enseñanza-aprendizaje (aula multigrado o no) muestra un patrón similar al observado con relación al área, lo que no debe sorprender ya que la educación multigrado tiende a concentrarse en las zonas rurales. Así, las brechas en puntos porcentuales también han tendido a duplicarse en el período observado, llegando a 26,5 puntos en 2014, cuando era de 14,2 puntos en 2007.

La figura 2 da cuenta de los índices de paridad relativos al porcentaje de estudiantes que logra desempeños satisfactorios en comprensión lectora. Aquí se muestra que las diferencias asociadas al sexo de los estudiantes (a favor de las niñas) se han mantenido con ligeras variaciones a lo largo del tiempo; es decir, las niñas han mostrado un porcen-

7 Nótese aquí en los demás análisis vinculados a la gestión que a lo largo de los últimos 15 años ha habido un proceso muy importante de migración de matrícula desde el sector estatal hacia el no estatal, de modo tal que hoy la matrícula no estatal en educación primaria representa un cuarto de la matrícula (25,2\%) cuando en 1999 representaba 12,3\% del total (Guadalupe et al., 2002, p. 68). Esta migración introduce mayores niveles de heterogeneidad en el sector no estatal y hace que las poblaciones no sean exactamente equivalentes: debido a la alta concentración de ingresos existente en el país, no es lo mismo un sector no estatal que atiende fundamentalmente al decil superior de ingresos del país que uno que atiende al cuartil superior.

8 Nótese que a partir de 2010 hay un mejor registro de la información sobre el área en la que se ubican las escuelas (antes de dicha fecha la información era autodeclarada y luego de esa fecha se define con base en la cartografía digital del Ministerio). En 2009, durante el tránsito a la nueva metodología de clasificación según área, se produjo una anomalía que ha sido notada en las publicaciones de resultados de la ECE hechas por el Ministerio de Educación (2012, p. 46) por lo que esos datos (2009) no deben considerarse para el análisis. Asimismo, debe tomarse en cuenta el proceso de urbanización del país, lo que implica que las poblaciones no sean exactamente equivalentes a lo largo del tiempo, pues es posible asumir que aquellos que migran hacia las zonas urbanas no tienen el mismo perfil educativo que quienes no lo hacen. 


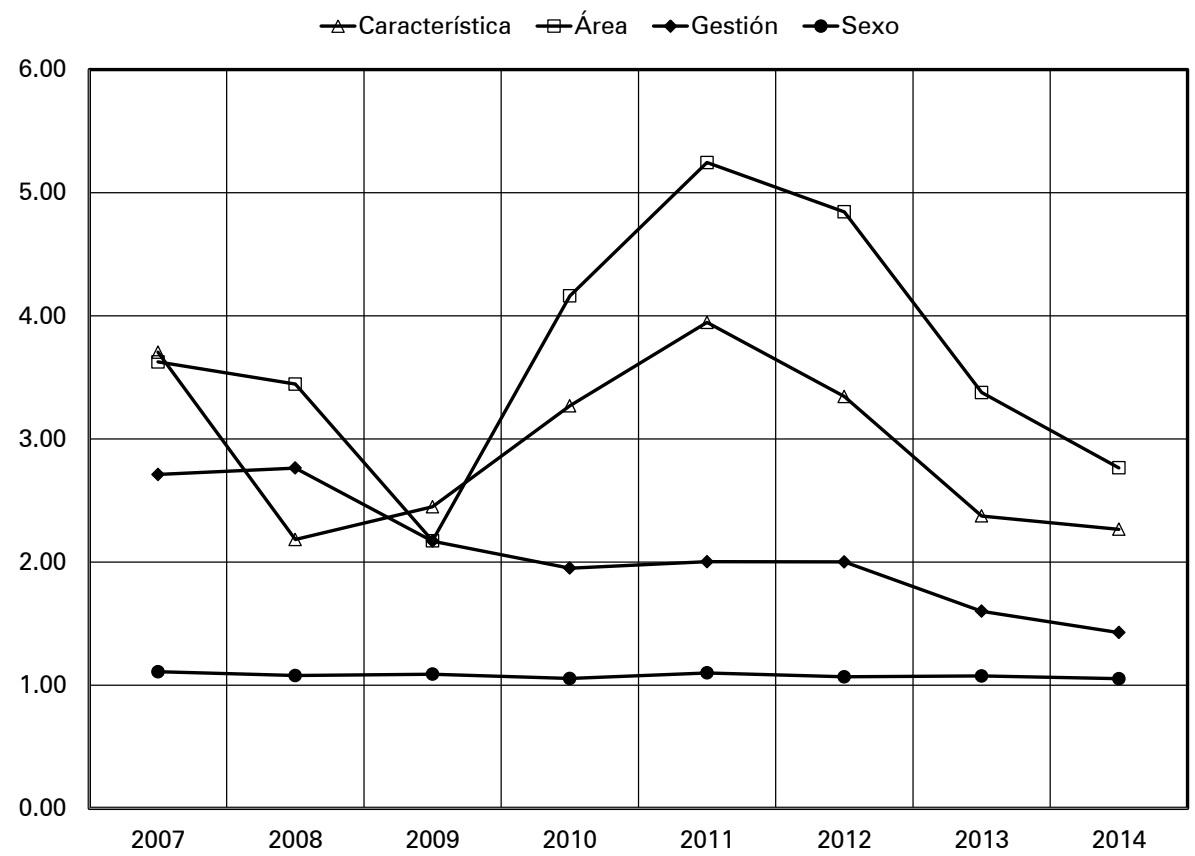

Figura 2. Índice de paridad entre los estudiantes de segundo grado que alcanzan el nivel de desempeño satisfactorio en comprensión lectora según estratos. Evaluación censal de estudiantes. Perú, 2007-2014.

Elaboración propia, a partir de la información disponible en http://umc.minedu. gob.pe/ (recuperada el 16 de marzo de 2015).

taje en el nivel satisfactorio entre $1,05 \mathrm{y}$ 1,11 veces mayor al de los niños.

Por su parte, el índice de paridad asociado a la gestión (favorable a la gestión no estatal) muestra un decrecimiento inicial importante entre 2007 y 2010, reduciéndose el índice de paridad de 2,7 a 2,0 (una reducción de 0,76 puntos o $28 \%$ del valor observado en 2007). Entre 2010 y 2012, la brecha se mantuvo constante, para luego reducirse hasta alcanzar un valor de 1,4 en 2014 (una reducción de 0,52 puntos o $27 \%$ del valor de 2010 ).
El índice de paridad por área (favorable a las áreas urbanas) muestra una tendencia inicial hacia la ampliación (pasando de 3,6 a 5,3 entre 2007 y 2011, lo que equivale a un incremento del $45 \%$ ), para luego experimentar una reducción de modo que el valor para 2014 fue de 2,8 (es decir, un $47 \%$ de reducción respecto del valor de 2011).

La información de acuerdo a la "característica" del proceso de enseñanza-aprendizaje (aula multigrado o no) 
muestra nuevamente un patrón similar al observado en el caso del área, aunque con valores menos pronunciados: el índice de paridad (a favor de las escuelas "completas") pasó de 3,7 en 2007 a 4,0 en 2011, para luego descender a 2,3 en 2014.

La figura 3 muestra los odds ratio relativos al porcentaje de estudiantes que logra desempeños satisfactorios en comprensión lectora. Las diferencias asociadas a sexo (a favor de las niñas) se han mantenido sin perturbaciones sig- nificativas. Se encuentra, así, que el $r a-$ tio ha oscilado entre 1,08 y 1,15 (en todo el periodo, las odds -o chances- de las niñas de estar en el nivel satisfactorio han sido en promedio $11 \%$ superiores al del caso de los niños).

El odds ratio asociado a la gestión (favorable a la gestión no estatal) muestra un aumento tenue entre el de 2007 y el de 2008 (de 3,54 a 3,74, es decir, un aumento de solo $6 \%$ ) para luego descender hasta el de 2010 (a 2,98). Entre el de 2010 y el de 2011 hubo un

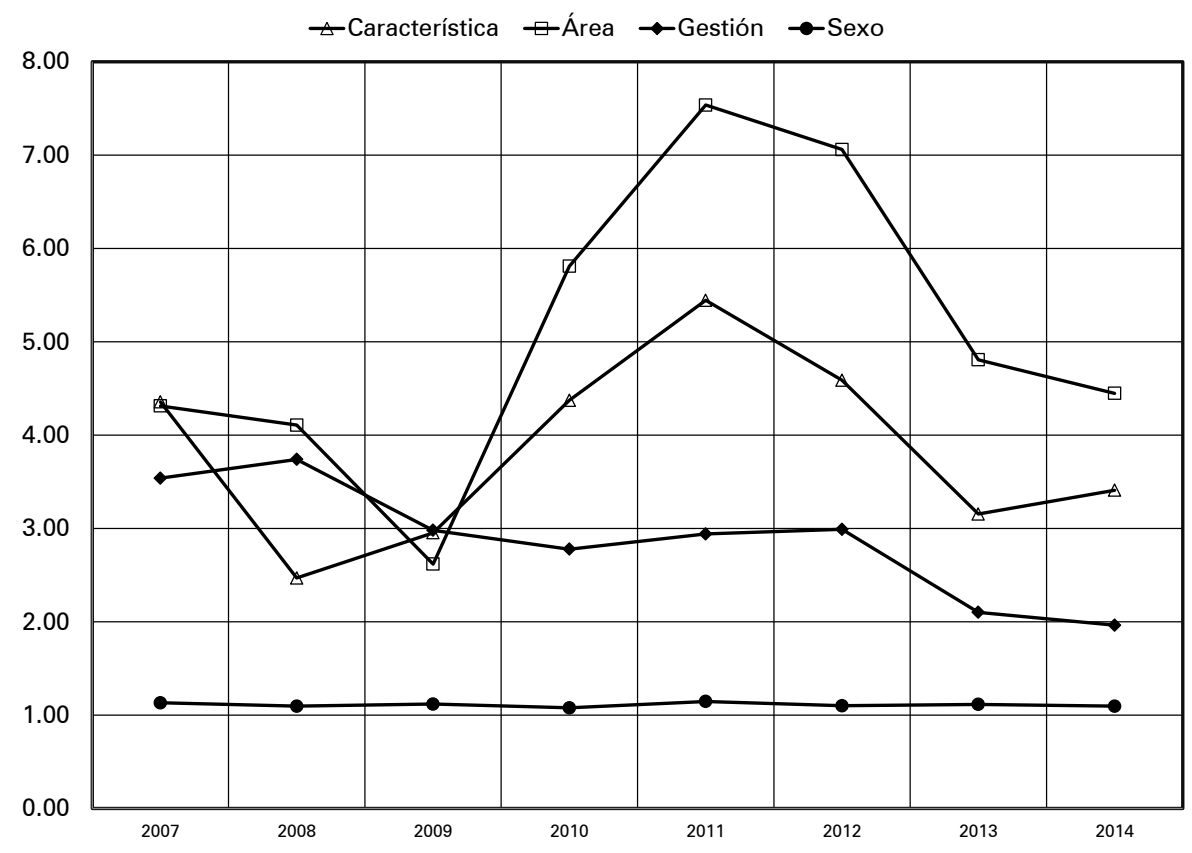

Figura 3. Odds ratio entre los estudiantes de segundo grado que alcanzan el nivel de desempeño satisfactorio en comprensión lectora según estratos. Evaluación censal de estudiantes. Perú, 2007-2014.

Elaboración propia, a partir de la información disponible en http://umc.minedu. gob.pe/ (recuperada el 16 de marzo de 2015). 
incremento poco pronunciado, y de allí se da una caída hasta llegar a 1,96 en el de 2014.

Mientras tanto, el odds ratio relacionado a área (a favor de áreas urbanas) muestra una tendencia inicial hacia la ampliación, pasando de 4,31 en 2007 a ser 7,54 veces en 2011 (un incremento de alrededor del $75 \%$ ). Luego, ha habido una reducción importante, llegando a 4,45 en 2014, aunque sin llegar al valor del de 2007.

Por último, el indicador relacionado con la característica del proceso de enseñanza-aprendizaje (a favor de escuelas multigrado), muestra un patrón similar a lo observado en el caso de área, pero más sutil. Solo cabría fijar la atención en que entre 2013 y 2014 hubo un ligero aumento (de 3,15 a 3,41), lo que se contradice con la tendencia a la reducción que se advirtió en el caso del área en la que se ubican las escuelas; además, este nivel se encuentra por debajo del que se poseía en 2007.

Como se mencionó al inicio de esta sección, se presenta dos tablas resumen, en las que se describe de modo sucinto la evolución de las brechas en los resultados de las evaluaciones censales de estudiantes entre 2007 y 2014. La primera muestra las brechas en el porcentaje de estudiantes con desempeño satisfactorio, tanto en comprensión lectora como en matemática, usando las tres medidas antes descritas; y la siguiente muestra las mismas medidas para el porcentaje de estudiantes con desempeño inicial en ambas pruebas.

Antes de presentar estos resultados, cabe reiterar que los valores del año 2009 para el caso de área son anomalías originadas en un error de clasificación de las escuelas según se detalla en la tabla 4. En este sentido, la descripción de la evolución de las brechas no considera estos valores.

Al comparar las brechas en comprensión lectora presentadas en la tabla 1 (desempeño satisfactorio) se tiene:

a) En el caso de las brechas según sexo, usando los tres indicadores considerados, muestran la misma tendencia: ligeras variaciones a lo largo del tiempo.

b) Respecto de las brechas por gestión, los tres indicadores no muestran la misma tendencia. La brecha absoluta señala una tendencia hacia la ampliación entre los años 2007 y 2012, para luego empezar a reducirse en el año 2014. Por su parte, el índice de paridad y el odds ratio muestran una tendencia a la reducción.

c) En cuanto al área, la brecha absoluta muestra una tendencia marcada a la ampliación; en cambio el índice de paridad y el odds ratio muestran una tendencia inicial a la ampliación entre los años 2007 y 2011, para luego reducirse. 
Tabla 1

Evolución de las brechas en los resultados de las evaluaciones censales de estudiantes. Porcentaje con desempeño satisfactorio. Perú, 2007-2014

\begin{tabular}{|c|c|c|c|}
\hline Estrato & Brecha absoluta & $\begin{array}{c}\text { Índice de paridad } \\
\text { Comprensión lectora }\end{array}$ & Odds ratio \\
\hline $\begin{array}{l}\text { Sexo } \\
\text { (favorable a } \\
\text { los niños) }\end{array}$ & $\begin{array}{l}\text { Tendencia tenue hacia la } \\
\text { ampliación (el valor en el } \\
2014 \text { llega a ser } 2,26 \\
\text { veces el de 2007). }\end{array}$ & $\begin{array}{l}\text { Ligeras variaciones a lo } \\
\text { largo del tiempo (entre } \\
1,05 \text { y } 1,11)\end{array}$ & $\begin{array}{l}\text { Ligeras variaciones a lo largo } \\
\text { del tiempo (entre } 1,08 \text { y } 1,15) \text {. }\end{array}$ \\
\hline $\begin{array}{l}\text { Gestión } \\
\text { (favorable a } \\
\text { estatal) }\end{array}$ & $\begin{array}{l}\text { Tendencia inicial a la } \\
\text { ampliación (2007- 2012) } \\
\text { para luego reducirse en } \\
\text { el } 2014 \text { por debajo del } \\
\text { nivel del } 2007 \text { (el valor } \\
\text { final llega a ser } 81 \% \text { del } \\
\text { valor inicial). }\end{array}$ & $\begin{array}{l}\text { Tendencia marcada hacia } \\
\text { la reducción (el valor de } \\
2014 \text { representa el } 53 \% \\
\text { del valor inicial de } 2007 \text { ). }\end{array}$ & $\begin{array}{l}\text { Tendencia hacia la reducción } \\
\text { (el ratio en } 2014 \text { representa el } \\
55 \% \text { del valor inicial de } 2007 \text { ). }\end{array}$ \\
\hline $\begin{array}{l}\text { Área } \\
\text { (favorable a } \\
\text { área rural) }\end{array}$ & $\begin{array}{l}\text { Tendencia marcada a la } \\
\text { ampliación (duplicó en el } \\
\text { periodo de análisis). }\end{array}$ & $\begin{array}{l}\text { Tendencia inicial hacia la } \\
\text { ampliación (2007 y 2011), } \\
\text { para luego reducirse por } \\
\text { debajo del nivel de } 2007 \\
\text { ( } 76 \% \text { del valor inicial). }\end{array}$ & $\begin{array}{l}\text { Tendencia inicial hacia la } \\
\text { ampliación (2007 y 2011), } \\
\text { para luego reducirse hasta } \\
\text { llegar cercanamente al nivel } \\
\text { de } 2007 .\end{array}$ \\
\hline \multirow[t]{2}{*}{$\begin{array}{l}\text { Característica } \\
\text { (favorable a } \\
\text { escuelas } \\
\text { multigrado } \\
\text { unidocentes) }\end{array}$} & $\begin{array}{l}\text { Tendencia marcada a la } \\
\text { ampliación (el valor de } \\
2014 \text { es } 1,87 \text { el valor } \\
\text { inicial de 2007). }\end{array}$ & $\begin{array}{l}\text { Tendencia inicial hacia la } \\
\text { ampliación ( } 2007 \text { y } 2011) \text {, } \\
\text { para luego reducirse por } \\
\text { debajo del nivel de } 2007 \\
\text { ( } 61 \% \text { del valor inicial). }\end{array}$ & $\begin{array}{l}\text { Tendencia inicial hacia la } \\
\text { ampliación ( } 2007 \text { y } 2011) \text {, } \\
\text { para luego reducirse hasta el } \\
\text { de } 2013 \text {. Entre } 2013 \text { y } 2014 \\
\text { hay una ampliación tenue, } \\
\text { pero el nivel permanece por } \\
\text { debajo del de } 2007 \text {. }\end{array}$ \\
\hline & \multicolumn{3}{|c|}{ Matemática } \\
\hline $\begin{array}{l}\text { Sexo } \\
\text { (favorable a } \\
\text { los niños) }\end{array}$ & $\begin{array}{l}\text { Incremento sostenido de } \\
\text { la brecha que pasa } \\
\text { de }-0,93 \text { en } 2007 \text { a }-6,55 \\
\text { en } 2014 \text {. }\end{array}$ & $\begin{array}{l}\text { Ligeras variaciones a lo } \\
\text { largo del tiempo (entre } \\
0,71 \text { y } 0,88)\end{array}$ & $\begin{array}{l}\text { Ligeras variaciones a lo largo } \\
\text { del tiempo (entre } 0,66 \text { y } 0,87 \text { ). }\end{array}$ \\
\hline $\begin{array}{l}\text { Gestión } \\
\text { (favorable a } \\
\text { estatal) }\end{array}$ & $\begin{array}{l}\text { Tendencia inicial hacia la } \\
\text { ampliación ( } 2007 \text { y } 2009) \\
\text { para luego reducirse y } \\
\text { ser estadísticamente } \\
\text { indistinguible de cero en } \\
2014 \text {. }\end{array}$ & $\begin{array}{l}\text { Aumento inicial tenue } \\
\text { ( } 2007 \text { y 2009), para luego } \\
\text { reducirse de forma } \\
\text { constante hasta ser } \\
\text { estadísticamente } \\
\text { indistinguible de uno en } \\
2014 \text {. }\end{array}$ & $\begin{array}{l}\text { Aumento inicial tenue ( } 2007 \text { y } \\
2009 \text { ), para luego reducirse de } \\
\text { forma constante hasta ser } \\
\text { estadísticamente indistinguible } \\
\text { de uno en } 2014 \text {. }\end{array}$ \\
\hline $\begin{array}{l}\text { Área } \\
\text { (favorable a } \\
\text { área rural) }\end{array}$ & $\begin{array}{l}\text { Tendencia marcada a la } \\
\text { ampliación (en } 2014 \text { es } \\
\text { casi cuatro veces el valor } \\
\text { de 2007). }\end{array}$ & $\begin{array}{l}\text { Tendencia inicial hacia la } \\
\text { ampliación (2007 y 2011), } \\
\text { para luego reducirse } \\
\text { hasta ser } 1,11 \text { veces el } \\
\text { valor de } 2007 .\end{array}$ & $\begin{array}{l}\text { Tendencia inicial hacia la } \\
\text { ampliación (2007 y } 2011) \text {, } \\
\text { para luego reducirse hasta ser } \\
1,27 \text { veces el valor de } 2007 \text {. }\end{array}$ \\
\hline $\begin{array}{l}\text { Característica } \\
\text { (favorable a } \\
\text { escuelas } \\
\text { multigrado } \\
\text { unidocentes) }\end{array}$ & $\begin{array}{l}\text { Tendencia marcada a la } \\
\text { ampliación (el valor de } \\
2014 \text { llega a ser } 4,37 \text { el } \\
\text { valor inicial de 2007). }\end{array}$ & $\begin{array}{l}\text { Tendencia inicial hacia la } \\
\text { ampliación ( } 2007 \text { y } 2011) \text {, } \\
\text { para luego reducirse } \\
\text { hasta ser } 1,23 \text { veces el } \\
\text { valor de } 2007 .\end{array}$ & $\begin{array}{l}\text { Tendencia inicial hacia la } \\
\text { ampliación (2007 y 2011), } \\
\text { para luego reducirse hasta el } \\
\text { de } 2013 \text {. Entre } 2013 \text { y } 2014 \\
\text { hay una ampliación tenue, y el } \\
\text { nivel final es } 1,42 \text { veces el } \\
\text { valor inicial del de } 2007 \text {. }\end{array}$ \\
\hline
\end{tabular}


d) Al considerar la característica de la escuela, la brecha absoluta muestra una tendencia marcada a la ampliación. El índice de paridad y el odds ratio muestran una tendencia inicial a la ampliación entre los años 2007 y 2011, para luego reducirse.

En resumen, en lo que se refiere al porcentaje de estudiantes en el nivel satisfactorio en comprensión lectora, solo en el caso de las brechas por sexo los tres indicadores muestran la misma tendencia. En general, hay mayor coincidencia entre el índice de paridad y el odds ratio.

Al comparar las brechas en matemática presentadas en la tabla 1 (desempeño satisfactorio) tenemos lo siguiente:

a) Las brechas según sexo muestran la misma tendencia en el caso del índice de paridad y el odds ratio: ligeras variaciones a lo largo del tiempo. Por su parte, la brecha absoluta muestra un incremento sostenido.

b) Al considerar las brechas por gestión, los tres indicadores presentan casi la misma tendencia, es decir, un aumento inicial tenue entre los años 2007 y 2009 , para luego perder significancia estadística en el año 2014.

c) En cuanto al área, tanto el índice de paridad como el odds ratio muestran una tendencia a la ampliación entre los años 2007 y 2011, para luego empezar a reducirse. Una tendencia diferente es mostrada por la brecha absoluta, la cual apunta hacia un aumento marcado.

d) Por último, la brecha según la característica de la escuela muestra una tendencia similar al considerar el índice de paridad y el odds ratio: se aprecia una ampliación entre los años 2007 y 2011, para luego empezar a reducirse. Al usar como indicador la brecha absoluta, esta muestra una tendencia marcada a la ampliación.

En resumen, en cuanto al porcentaje de estudiantes en el nivel satisfactorio en matemática, solo en el caso de las brechas por gestión los tres indicadores muestran una tendencia similar. Asimismo, como en el caso anterior, hay mayor coincidencia entre el índice de paridad y el odds ratio.

Al comparar las brechas en comprensión lectora presentadas en la tabla 2 (desempeño satisfactorio) se tiene:

a) Las brechas según sexo muestran la misma tendencia, es decir, ligeras variaciones a lo largo del tiempo.

b) En el caso de las brechas por gestión, los tres indicadores no muestran la misma tendencia. La brecha absoluta apunta hacia la reducción, mientras que el índice de paridad y el odds ratio muestran, de un modo análogo, una tendencia a la ampliación entre los años 2007 y 2012, 
para luego constatar una reducción de la brecha.

c) En cuanto al área, tanto la brecha absoluta como el odds ratio muestran una tendencia a la ampliación entre los años 2007 y 2011, para luego verificarse una reducción de la brecha. En cambio, el índice de paridad muestra una tendencia marcada hacia la ampliación.

d) Finalmente, al considerar la característica de las escuelas, tanto la brecha absoluta como el odds ratio muestran una tendencia a la ampliación entre los años 2007 y 2011, para luego dar cuenta de una reducción de la brecha. Por su parte, el índice de paridad muestra una tendencia marcada hacia la ampliación.

En resumen, en lo que respecta al porcentaje de estudiantes en el nivel inicial en comprensión lectora, solo en el caso de las brechas por sexo los tres indicadores muestran la misma tendencia. Asimismo, en general se observa una mayor coincidencia entre la brecha absoluta y el odds ratio.

En cuanto al porcentaje de estudiantes en el nivel inicial en matemática, también mostrado en la tabla 2 , se observa lo siguiente:

a) Las brechas según sexo muestran la misma tendencia en los tres indica- dores. Hay una reducción fuerte de la brecha entre los años 2007 y 2009, para luego presentar cierta estabilidad.

b) Al considerar las brechas por gestión, los tres indicadores presentan la misma tendencia. A saber, ligeras variaciones entre los años 2007 y 2009, para luego desaparecer las brechas.

c) En cuanto al área, tanto la brecha absoluta como el odds ratio exhiben una tendencia a la ampliación entre los años 2007 y 2011, para luego mostrar una reducción de la brecha. Por el contrario, el índice de paridad presenta una tendencia marcada hacia la ampliación.

d) Finalmente, al considerar la característica de las escuelas, tanto la brecha absoluta como el odds ratio muestran una tendencia a la ampliación entre los años 2007 y 2011, para luego tender a la reducción de la brecha. El índice de paridad presenta una tendencia marcada hacia la ampliación.

Resumiendo la información correspondiente al porcentaje de estudiantes en el nivel de inicio en matemática, en el caso de las brechas por sexo y gestión los tres indicadores muestran la misma tendencia. En general hay mayor coincidencia entre la brecha absoluta y el odds ratio. 
Tabla 2

Evolución de las brechas en los resultados de las evaluaciones censales de estudiantes. Porcentaje con desempeño en nivel inicial. Perú, 2007-2014

\begin{tabular}{|c|c|c|c|}
\hline Estrato & Brecha absoluta & $\begin{array}{c}\text { Índice de paridad } \\
\text { Comprensión lectora }\end{array}$ & Odds ratio \\
\hline $\begin{array}{l}\text { Sexo } \\
\text { (favorable a } \\
\text { los niños) }\end{array}$ & $\begin{array}{l}\text { Ligeras variaciones a lo } \\
\text { largo del tiempo entre } \\
\text { cero y } 1,20 \text { puntos } \\
\text { porcentuales. }\end{array}$ & $\begin{array}{l}\text { Ligeras variaciones a lo } \\
\text { largo del tiempo (entre } \\
\text { uno y } 1,09 \text { puntos } \\
\text { porcentuales). }\end{array}$ & $\begin{array}{l}\text { Ligeras variaciones a lo } \\
\text { largo del tiempo (entre } \\
\text { uno y } 1,10 \text { puntos } \\
\text { porcentuales). }\end{array}$ \\
\hline $\begin{array}{l}\text { Gestión } \\
\text { (favorable a } \\
\text { estatal) }\end{array}$ & $\begin{array}{l}\text { Tendencia marcada } \\
\text { hacia la reducción (el } \\
\text { valor de } 2014 \text { es } 42 \% \text { el } \\
\text { valor inicial de } 2007 \text { ) }\end{array}$ & $\begin{array}{l}\text { Tendencia inicial hacia } \\
\text { la ampliación ( } 2007 \text { y } \\
2012 \text { ), para luego } \\
\text { reducirse por debajo del } \\
\text { nivel de } 2007 \text { (el valor } \\
\text { de } 2014 \text { representa } \\
\text { el } 88 \% \text { del de } 2007 \text { ). }\end{array}$ & $\begin{array}{l}\text { Tendencia inicial hacia } \\
\text { la ampliación ( } 2007 \text { y } \\
2012 \text { ), para luego } \\
\text { reducirse por debajo del } \\
\text { nivel de } 2007 \text { (el valor } \\
\text { de } 2014 \text { representa } \\
\text { el } 71 \% \text { del de } 2007 \text { ). }\end{array}$ \\
\hline $\begin{array}{l}\text { Área } \\
\text { (favorable a } \\
\text { área rural) }\end{array}$ & $\begin{array}{l}\text { Tendencia inicial hacia } \\
\text { la ampliación ( } 2007 \text { y } \\
2011) \text {, para luego } \\
\text { reducirse por debajo del } \\
\text { nivel de } 2007 \text { (llega a } \\
\text { ser } 75 \% \text { del valor } \\
\text { inicial). }\end{array}$ & $\begin{array}{l}\text { Tendencia marcada } \\
\text { hacia la ampliación (el } \\
\text { valor de } 2014 \text { es } 1,52 \\
\text { el de } 2007) .\end{array}$ & $\begin{array}{l}\text { Tendencia inicial hacia } \\
\text { la ampliación ( } 2007 \text { y } \\
2011) \text {, para luego } \\
\text { reducirse hasta el de } \\
2014 \text { llegando a ser } 1,23 \\
\text { veces el valor de } 2007 \text {. }\end{array}$ \\
\hline $\begin{array}{l}\text { Característica } \\
\text { (favorable a } \\
\text { escuelas } \\
\text { multigrado } \\
\text { unidocentes) }\end{array}$ & $\begin{array}{l}\text { Tendencia hacia la } \\
\text { ampliación ( } 2007 \text { y } \\
2011) \text {, para luego } \\
\text { reducirse por debajo del } \\
\text { nivel de } 2007 \text { (llega a } \\
\text { ser } 69 \% \text { del valor } \\
\text { inicial). }\end{array}$ & $\begin{array}{l}\text { Tendencia marcada } \\
\text { hacia la ampliación } \\
\text { (el valor de } 2014 \text { es } \\
1,43 \text { veces el de } 2007 \text { ). }\end{array}$ & $\begin{array}{l}\text { Tendencia inicial hacia } \\
\text { la ampliación ( } 2007 \text { y } \\
2011) \text {, para luego } \\
\text { reducirse hasta el de } \\
2013 \text {. Entre } 2013 \text { y } 2014 \\
\text { hay una ampliación } \\
\text { tenue, llegando a ser } \\
1,09 \text { veces el valor de } \\
2007 \text {. }\end{array}$ \\
\hline
\end{tabular}

Matemática

\begin{tabular}{|c|c|c|c|}
\hline $\begin{array}{l}\text { Sexo } \\
\text { (favorable a } \\
\text { los niños) }\end{array}$ & $\begin{array}{l}\text { Reducción fuerte entre } \\
2007 \text { y } 2009, \text { para luego } \\
\text { mostrar cierta } \\
\text { estabilidad por encima } \\
\text { del valor del de } 2009 \\
\text { (pasa de ser }-0,45 \text { en } \\
2007 \text { a }-3,41 \text { en } 2014 \text { ). }\end{array}$ & $\begin{array}{l}\text { Reducción fuerte entre } \\
2007 \text { y } 2009 \text {, para luego } \\
\text { mostrar cierta } \\
\text { estabilidad por encima } \\
\text { del valor de } 2009 \text { (el } \\
\text { valor de } 2014 \text { es } 92 \% \\
\text { del valor de } 2007 \text { ). }\end{array}$ & $\begin{array}{l}\text { Reducción fuerte entre } \\
2007 \text { y } 2009, \text { para luego } \\
\text { mostrar cierta } \\
\text { estabilidad por encima } \\
\text { del valor de } 2009 \text { (el } \\
\text { valor de } 2014 \text { es } 88 \% \\
\text { del valor de 2007). }\end{array}$ \\
\hline $\begin{array}{l}\text { Gestión } \\
\text { (favorable a } \\
\text { estatal) }\end{array}$ & $\begin{array}{l}\text { Ligeras variaciones } \\
\text { entre } 2007 \text { y } 2009 \text { para } \\
\text { luego decrecer y ser } \\
\text { estadísticamente } \\
\text { indistinguible de cero en } \\
2014 \text {. }\end{array}$ & $\begin{array}{l}\text { Ligeras variaciones } \\
\text { entre } 2007 \text { y } 2009 \text { para } \\
\text { luego reducirse hasta } \\
\text { ser estadísticamente } \\
\text { indistinguible de uno en } \\
2014 \text {. }\end{array}$ & $\begin{array}{l}\text { Ligeras variaciones } \\
\text { entre } 2007 \text { y } 2009 \text { para } \\
\text { luego reducirse hasta } \\
\text { ser estadísticamente } \\
\text { indistinguible de uno en } \\
2014 \text {. }\end{array}$ \\
\hline $\begin{array}{l}\text { Área } \\
\text { (favorable a } \\
\text { área rural) }\end{array}$ & $\begin{array}{l}\text { Tendencia inicial hacia } \\
\text { la ampliación ( } 2007 \text { y } \\
2011) \text {, para luego } \\
\text { reducirse sin llegar a los } \\
\text { niveles de } 2007 \text { (1,69 } \\
\text { veces más en } 2014 \text { que } \\
\text { en 2007). }\end{array}$ & $\begin{array}{l}\text { Tendencia marcada } \\
\text { hacia la ampliación, } \\
\text { siendo el valor de } 2014 \\
1,32 \text { veces que en } 2007 \text {. }\end{array}$ & $\begin{array}{l}\text { Tendencia inicial hacia } \\
\text { la ampliación ( } 2007 \text { y } \\
2011) \text {, para luego } \\
\text { reducirse sin llegar a los } \\
\text { niveles de } 2007(1,46 \\
\text { veces más en } 2014 \text { que } \\
\text { en 2007). }\end{array}$ \\
\hline
\end{tabular}




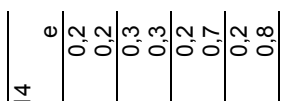

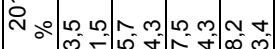

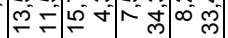

- m) m m n n

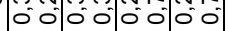

$m$

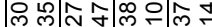

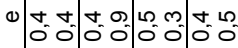
กิ

茫

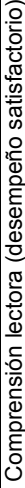

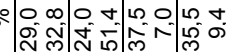

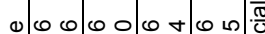

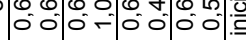
它 응

- Nิ

- 1 م $\begin{array}{llllllll}0 & 0 & 0 & -1 & 0 & 0 & 0 & 0\end{array}$ ⿶)을

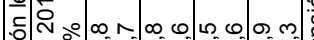

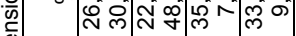

:은을

$\circ$.

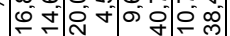

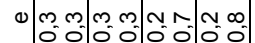

is

¿

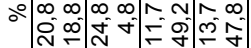

๑ 0.000000

ำ

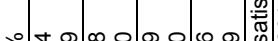

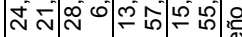

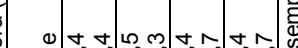

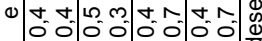

(

䔅응

Ã

O

응

คิ.

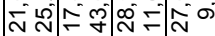

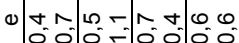

$\infty$

g.०.hnanotrom

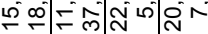

- :- :

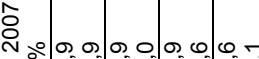

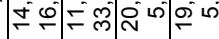

8

$\frac{\pi}{2} \frac{2}{2}$

ก

ฏิ ปิ

ว ข

5

:

$\pi$ m 0

$\frac{\sqrt{6}}{\frac{10}{6}}$

$\frac{0}{0} \frac{\sqrt{0}}{0}$

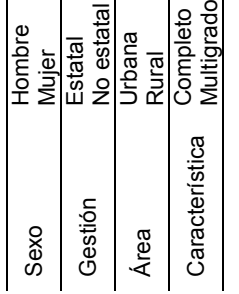

(1) م

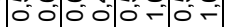

\%

i. ma or. $00.0 m$

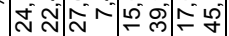

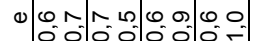

$\infty$

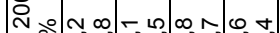

m

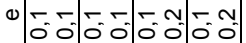

容

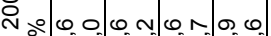

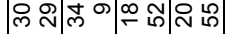

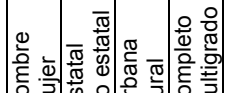

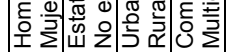

:드유.

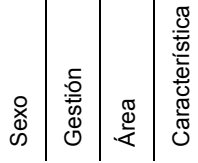

:음

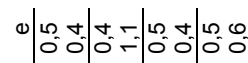

蕰

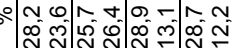

(1) o. 0 0. 000

m

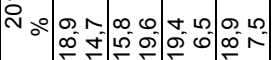

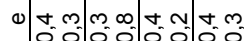

ำ

i... $-4 \operatorname{los} n-00$

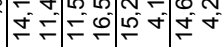

- 1 L

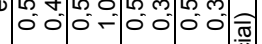

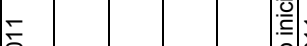

$\sqrt{2}$

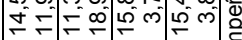

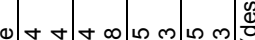

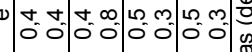

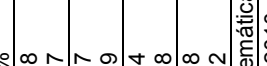
管

ษ $N=0^{\circ}$

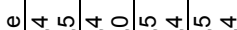

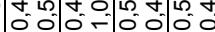

8)

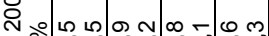

ป พิำ

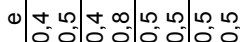

$\infty$

穴

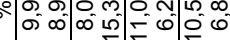

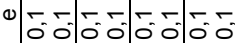

○ิ

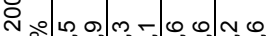

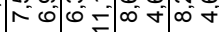

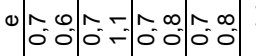

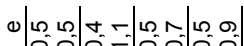

更

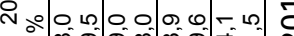

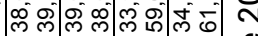

-

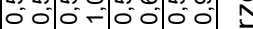

m है

No r m m tam a

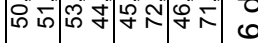

(1)

00000000

ㄴ.

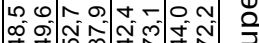

t大

20

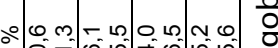

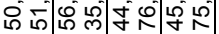

- on OnNa

$00=0$ - 0 - 0

은

$\infty \infty 0-00 .+\infty$

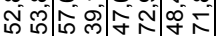

- N $\infty$ N 0 - 0 - 0

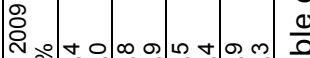

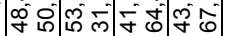

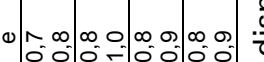

$\infty$ :

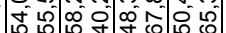

$0 .--6-n-n$

o, $0,0,0,0,0,0$

\& $\quad \frac{0}{0}$

in

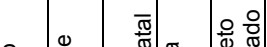

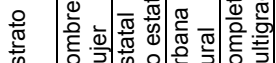

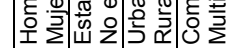

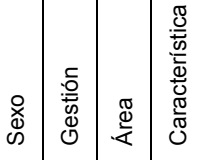

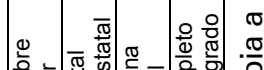

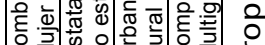

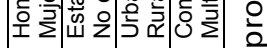

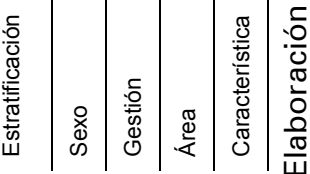




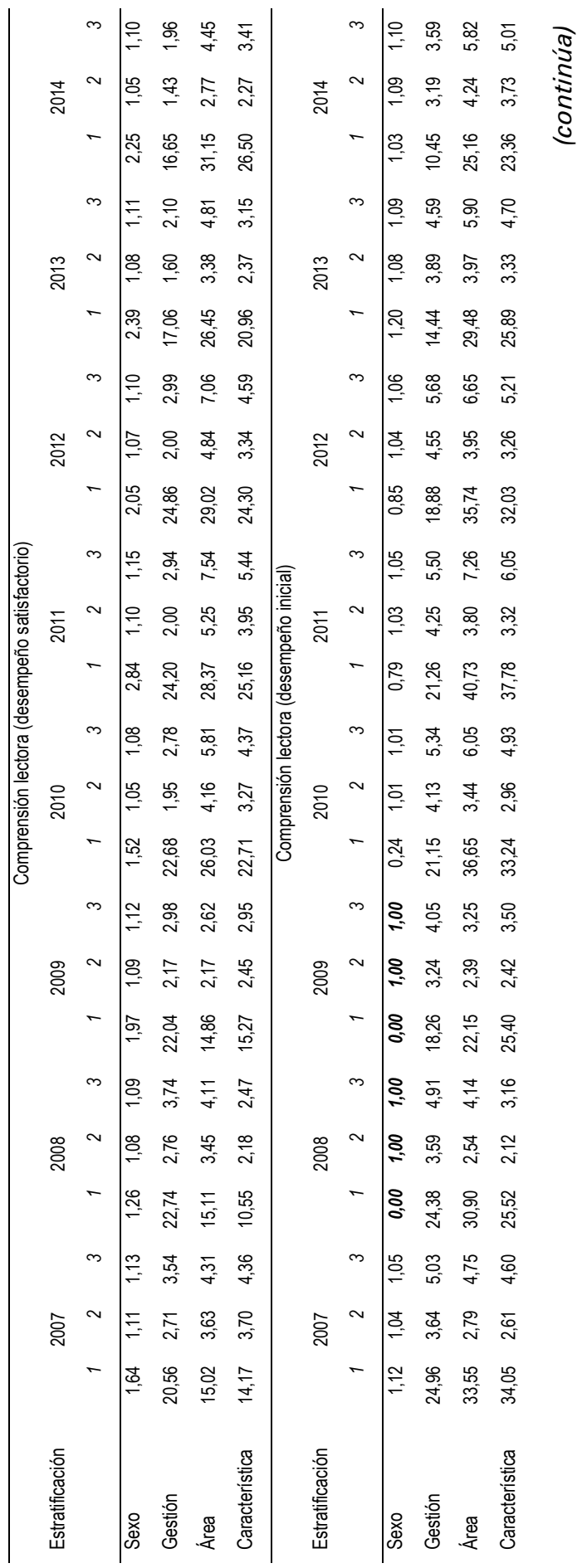




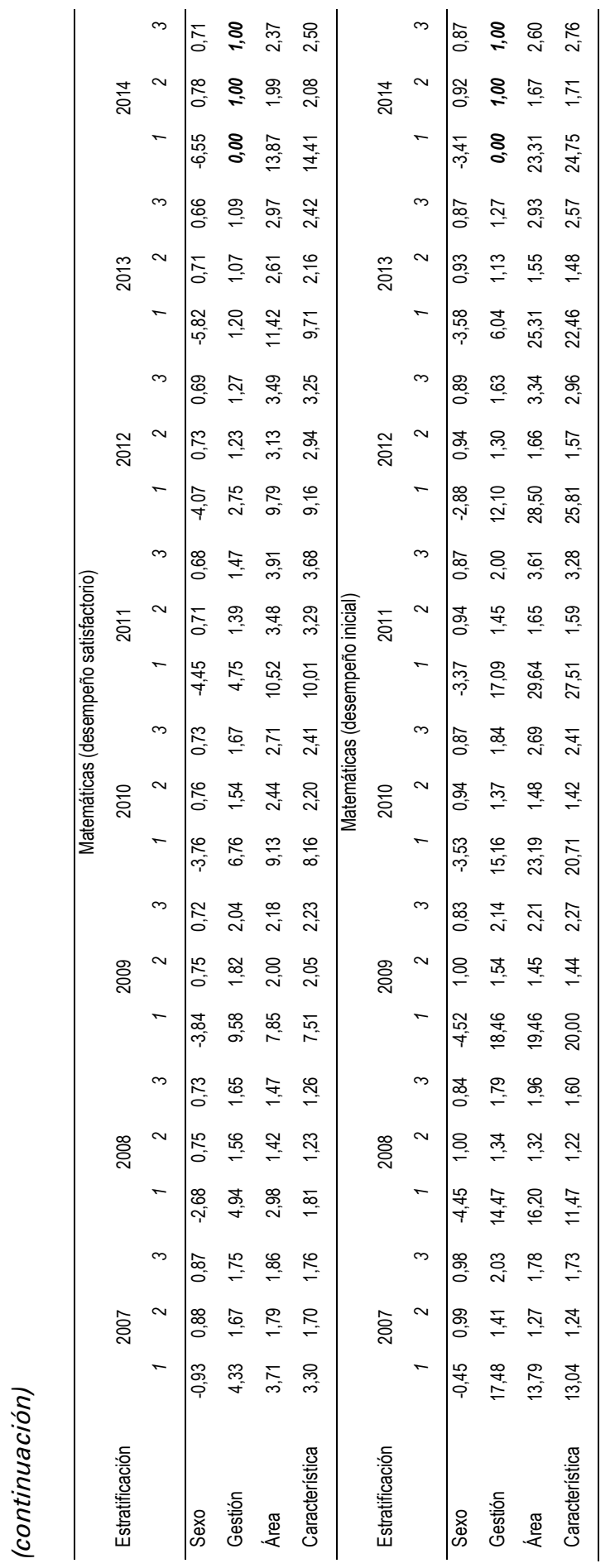

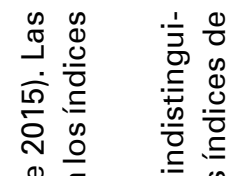

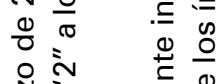

ำ 닐

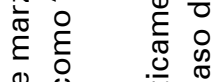

$\begin{array}{llll}0 & 0 & 0 & \pi \\ 0 & 0 & 0 \\ 0 & 0 & 0 & 0 \\ 0 & 0 & 0 & 0 \\ 0 & 0 & 0 & 0\end{array}$

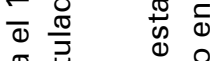

苋 인 \&

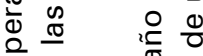

号

仓 $\frac{\Phi}{0} \quad 0 \frac{0}{0}$

ఏ

웡

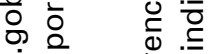

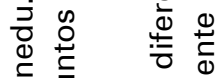

ह气 ڤ

एँ

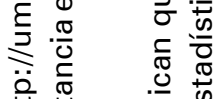

鱼恋

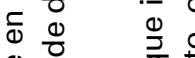

응 중. 후음

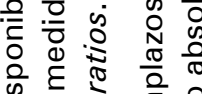

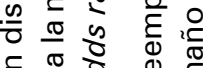

등

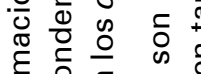

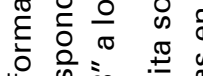

일 के

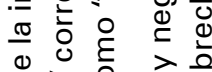

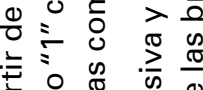

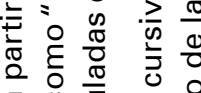

일

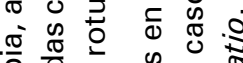

응 क \& क

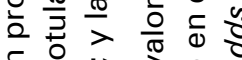

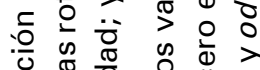

๘ั0

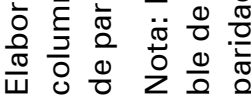




\section{ComentaRIOS FinALES}

Los resultados que anualmente genera la evaluación censal de estudiantes desde 2007, han mostrado, de modo sistemático, una tendencia general hacia cada vez mayores niveles de desempeño entre los estudiantes del país. Estos resultados son consistentes con la información que ha sido generada también a través de otras evaluaciones estandarizadas de estudiantes, como es el caso de los dos estudios latinoamericanos conducidos en 2006 y 2013 (Unesco/Santiago, 2014) así como del Programme for International Student Assessment (PISA) de la OCDE (Ministerio de Educación, 2013) en el que participó el Perú en 2001, 2009 y 2012.

Ahora bien, constatar este progreso sostenido no significa olvidar que los niveles de desempeño que logran los estudiantes peruanos son aún pobres y existen importantes disparidades que los desempeños medios, por su propia naturaleza, no muestran. Justamente, este artículo busca dar una mirada sistemática a dichas disparidades.

Una observación detenida a las brechas existentes supone ser cuidadosos respecto del enfoque metodológico que se adopte. En este artículo se han tenido en cuenta tres métodos y se ha optado por observar las disparidades en los dos extremos de la distribución de desempeños: entre aquellos que logran un desempeño considerado satisfactorio, y aquellos que solo muestran un desempeño inicial (el nivel más bajo identificado por la ECE).
Al observar estas diversas medidas se constata que los ritmos de crecimiento del desempeño satisfactorio y de reducción del desempeño inicial son mayores entre los grupos más rezagados en el último período, ya que, en general, los primeros años de la serie muestran una tendencia al incremento de las brechas.

Si bien no se cuenta con información suficiente para explicar de modo riguroso este comportamiento, es muy probable que la consolidación de la ECE como práctica regular y de alta visibilidad haya jugado un papel en este proceso. Alguna evidencia sugiere (Guadalupe y Castillo, 2014) que la presencia regular y sostenida de la ECE ha motivado diferentes reacciones de los actores educativos, las que pueden traducirse en modificaciones en los patrones de resultados, especialmente en la medida en que la ECE se consolida; así, los años iniciales de la serie pueden mostrar una tendencia diferente de aquella observada en los años más recientes. Ahora bien, no todas esas reacciones son necesariamente positivas, ya que hay casos en los que se sobreenfatiza la importancia de afectar el indicador (los resultados en la ECE) por encima de lograr las metas (asegurar aprendizajes). Confundir la meta sustantiva con el indicador es una forma muy fácil de generar resultados no deseables.

La brecha que muestra cambios más marcados hacia el cierre es la correspondiente a la gestión de las escuelas. Es 
importante notar que dicha tendencia al cierre de brechas se produce en un período en el que la matrícula no estatal (sobre todo la parte correspondiente a la educación privada pagada) ha tendido a expandirse de modo sostenido, tanto por su mayor capacidad para absorber nuevas matrículas como por la migración de estas desde el sector estatal (particularmente desde los colegios gestionados por el sector Educación). Esto ha llevado a que la educación no estatal sea hoy más heterogénea de lo que solía ser cuando su oferta se concentraba en un sector menor de la población. Esta heterogeneidad se traduce en la presencia de un gran número de escuelas privadas pagadas que no aseguran aprendizajes básicos a sus estudiantes, lo que sugiere que hay otros factores valorados por los padres (¿seguridad, jornada extendida, valor simbólico - no estigma?) que explican la decisión de optar por una escuela privada.

Por su parte, el comportamiento de las brechas según área (urbana/rural) y característica de la escuela (completa/ multigrado) muestran una gran proximidad y un patrón de agudización, primero, y tendencia al cierre después.

Haber usado diferentes métodos para medir brechas muestra que las labores de análisis pueden optar por distintas posibilidades y tener distintos derroteros que hay que manejar con atención y prudencia. Las diversas medidas pueden derivar en múltiples historias o relatos, por lo que es menester tener claro que ninguna es intrínsecamente mejor que las otras, sino que cada una sirve para destacar otros aspectos o enfatizar matices que son propios de un fenómeno complejo: las brechas en puntos porcentuales dan cuenta de una distancia, pero no permiten captar los diversos ritmos de cambio ni la dificultad relativa de incrementar un punto porcentual, dependiendo del punto de la escala en el que se encuentra (diferentes esfuerzos marginales); las diferencias relativas dan cuenta del tamaño relativo de las brechas; mientras que los odds ratio nos muestran las diferencias en ratios de probabilidad que, por lo mismo, brindan una medida que combina tanto una posición relativa como las distancias absolutas.

\section{RefERENCIAS}

A'Court, C., Stevens, R., y Heneghan, C. (2012). Against all odds? Improving the understanding of risk reporting. The British Journal of General Practice: The Journal of the Royal College of General Practitioners, 62(596),220-223.doi:10.3399/ bjgp12X630223.

Bond, T., y Fox, C. (2001). Applying the Rasch model fundamental measurement in the human sciences. Mahwah, N.J.: L. Erlbaum.

Coleman, J. S., Campbell, E. Q., Hobson, C. J., McPartland, F., Mood, A. M., y Weinfeld, F. D. (1966). Equality of educational opportunity. Washington DC: US Government Printing Office. 
Cueto, S., Guerrero, G., León, J., Zapata, M., y Freire, S. (2013). ¿La cuna marca las oportunidades y el rendimiento educativo? Una mirada al caso peruano. Lima: Grade.

Guadalupe, C., y Castillo, L. E. (2014). Diferencias regionales en políticas de apoyo al aprendizaje y su posible impacto sobre los niveles de logro estudiantil (N. ${ }^{\circ}$ DD1412). Lima. Recuperado de http://www. up.edu.pe/ciup/discusionDetalle. aspx?idDet $=3634$

Guadalupe, C., y Villanueva, A. (2013). PISA 2009/2000 en América Latina: una relectura de los cambios en el desempeño lector y su relación con las condiciones sociales. Apuntes, XL(72), 157-192. Recuperado de http://revistas.up.edu.pe/index.php/ apuntes/article/view/22/17

Guadalupe, C., Huillcamisa, J., Miranda, L., Quintana, M. L., Rodríguez, J., Santillán, N., Silva, J., y Zambrano, G. (2002). La educación peruana al inicio del nuevo siglo. Lima: Ministerio de Educación. Recuperado de http://umc.minedu.gob.pe/?p=381

Kline, R. (2004). Beyond significance testing: reforming data analysis methods in behavioral research. Washington DC: American Psychological Association.

Ministerio de Educación. (2005). IV Evaluación Nacional del Rendimiento Estudiantil 2004. Informe descriptivo de resultados. Documento de Trabajo UMC. (N.. 12). Lima: Ministerio de Educación.

Ministerio de Educación. (2012). Resultados de la Evaluación Censal de Estudiantes 2011 (ECE 2011)Presentación. Lima. Recuperado de http://www2.minedu.gob.pe/umc/ ece2011/Presentaciones/PPTECE2011UMC.ppt

Ministerio de Educación. (2013). PISA 2012: Primeros resultados. Informe nacional del Perú. Lima: Ministerio de Educación. Recuperado de http:// www2.minedu.gob.pe/umc/PISA/ Pisa2012/Informes_de_resultados/ LibroPisa CompletoPDF.pdf

Sistrom, C. L., y Garvan, C. W. (2004). Proportions, odds, and risk. Radiology, 230(1), 12-19. doi:10.1148/radiol.2301031028.

Szumilas, M. (2010). Explaining odds ratios. Journal of the Canadian Academy of Child and Adolescent Psychiatry $=$ Journal de l'Académie Canadienne de Psychiatrie de L'enfant et de L'adolescent, 19(3), 227-229.

Unesco/Santiago. (2007). Educación de calidad para todos: un asunto de derechos humanos. Santiago de Chile: Unesco. Recuperado de http://unesdoc.unesco.org/images/ 0015/001502/150272s.pdf.

Unesco/Santiago. (2014). TERCE. Primera entrega de resultados. Santiago de Chile: Unesco. Recuperado de https:// docs.google.com/file//0BwzG1KOQ MVTIX3Zxb3NxeEN0cXc/edit 
Willms, D. (2002). Ten hypotheses about socioeconomic gradients and community differences in children's developmental outcomes. Hull, Quebec: HRCD.
Wright, B., y Linacre, J. (1987). Dichotomous Rasch model derived from objectivity. En J. Linacre (Ed.), Rasch measurement transactions. Part 1 (pp. 5-6). Chicago, Il: MESA Press. 